\title{
SYNTHESIS AND NUCLEAR MAGNETIC RELAXATION PROPERTIES OF COMPOSITE IRON OXIDE NANOPARTICLES
}

\author{
Elisa M. N. de Oliveira ${ }^{\mathrm{a}, \mathrm{b}, *, \mathbb{D}}$, Maximiliano S. da Rocha ${ }^{\mathrm{a}}$, Ana Paula P. Froner ${ }^{\mathrm{c}}$, Nara R. S. Basso ${ }^{\mathrm{a}, \mathrm{b}}$, Mara L. Zanini ${ }^{\mathrm{b}}$ and $^{-}$ \\ Ricardo M. Papaléo ${ }^{\mathrm{a}, \mathrm{b}}$ \\ ${ }^{a}$ Centro Interdisciplinar de Nanociência e Micro-Nanotecnologia, Pontifícia Universidade Católica do Rio Grande do Sul, 90619 - \\ 900 Porto Alegre - RS, Brasil

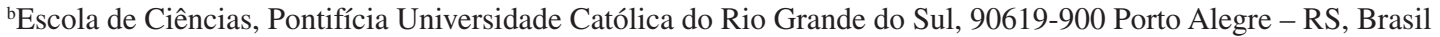 \\ ${ }^{c}$ Hospital São Lucas, Pontifícia Universidade Católica do Rio Grande do Sul, 90619-900 Porto Alegre - RS, Brasil
}

Recebido em 11/06/2018; aceito em 20/09/2018; publicado na web em 14/11/2018

\begin{abstract}
Superparamagnetic iron oxide nanoparticles are suitable contrast agents for magnetic resonance imaging due to their effect on proton relaxation times, and excellent biocompatibility and biodistribution in tissue. In the present study, a ferrofluid formed by dextrancoated iron oxide nanoparticles was prepared by the coprecipitation method and with subsequent steps of crosslinking and addition of functionalizations on the nanoparticle's surface (amino groups and a fluorophore). The effect of differences in the synthesis route (ratio of dextran to iron salts and temperature of the reaction) on the physico-chemical characteristics of the nanoparticles and their performance as contrast agents for magnetic resonance imaging was investigated. Overall, the nuclear magnetic relaxation times of water protons in aqueous suspensions of the nanoparticles did not show major differences among different batches, with no significant effect due to the addition of the fluorophore or changes in the dextran fraction of up to $50 \%$. Larger reductions in the ratio of dextran to iron salts resulted in particles with slightly larger sizes, higher saturation magnetization and distinct nuclear magnetic relaxation times.
\end{abstract}

Keywords: nanoparticles; biomedical imaging; contrast agents.

\section{INTRODUCTION}

Ferrofluids are colloidal suspensions, consisting of nanometersized magnetic particles suspended in a liquid medium, which are finding a growing number of biomedical applications. One of the first methods of synthesis of magnetic particles in the presence of a solvent and a surfactant was proposed by Khalafalla, which produced ferrofluids stabilized by the addition of an oleic acid coating. ${ }^{1}$ Since then, several other methods have been used to synthesize these nanoparticles, such as microemulsion and sol-gel, ${ }^{2}$ electrospray, ${ }^{3}$ thermal decomposition, ${ }^{4}$ sonolysis, ${ }^{5}$ and coprecipitation. ${ }^{6-10}$ In the case of iron oxide nanoparticles (IONP), the synthesis by coprecipitation is one of the simplest and most efficient methods of production, involving reactions at low temperatures and relatively short reaction times. In addition, it has already proven to be an economically viable route, allowing large scale production. ${ }^{11}$

In the biomedical area, iron oxide nanoparticles can be used for in vitro applications, such as separation and labeling of biomolecules, cells, DNA/RNA and microorganisms. ${ }^{12}$ They can also be used in a variety of in vivo applications, including medical imaging, drug delivery and cancer treatment. ${ }^{11,13,14}$ In particular, the superparamagnetic behavior of sufficiently small particles favors their use as contrast agents for magnetic resonance imaging (MRI), ${ }^{15,16}$ being together with the complexes of gadolinium, the most common contrast agents for MRI. ${ }^{17}$ Iron oxide nanoparticles, when injected into the body, create an additional local magnetic field, which increases the transverse relaxation rate of water protons in the tissues and reduces the signal strength, producing a negative (dark) contrast in the regions they are located. ${ }^{16,18}$

Nanoparticles for in vivo applications should not only be stable, biocompatible and non-toxic, but also have an appropriate surface chemistry and size distribution. Therefore, the stabilization

*e-mail: elisa.oliveira@acad.pucrs.br of iron oxide nanoparticles with suitable coatings is essential to obtain a system, which is stable against aggregation, particularly in biological environments, and under the action of a magnetic field. ${ }^{19-21}$ Since their introduction as contrast agents for MRI in 1987, contrast agents based on iron oxide nanoparticles have been produced with different biocompatible coatings and with the addition of vector molecules with specificity to various diseases..$^{22,23}$ To inhibit the process of encapsulation by macrophages in living systems and increase blood circulation times, the nanoparticles can be coated with hydrophilic and biocompatible molecules, such as chitosan, ${ }^{24-26}$ dextran, ${ }^{21,27-31}$ carboximetildextran (DCM), ${ }^{32,33}$ or polyethelyne glycol, ${ }^{34,35}$ which may be inserted during or after the synthesis. In particular, dextran-coated superparamagnetic iron oxide nanoparticles (IONP-DX) is an important platform for the synthesis of multifunctional imaging agents. ${ }^{11,14,20}$ In order to further stabilize the IONP-DX, the organic coating can be reacted with a crosslinking agent (e.g., epichlorohydrin) that binds the partly free floating dextran chains around the iron core and forms a crosslinked network, encaging the iron oxide core. In one type of platform, labelled CLIO- $\mathrm{NH}_{2}$, nucleophilic amino groups are also added on the crosslinked surface for further chemical conjugation. ${ }^{36}$ Fluorophores may also be added to combine optical and MRI imaging capabilities, complementary techniques widely used in clinical and pre-clinical investigations. ${ }^{37,38}$ In this respect, fluorophores, like the Cy5.5 dye which emit in the near-infrared (NIR), are specially useful due to the larger penetration of light in this spectral range in biological tissues. ${ }^{39-42}$

In this work, we report the synthesis of composite nanoparticles with a magnetic iron oxide core coated with dextran, focusing on the impact of changes in the synthesis parameters (the mass ratio of polysaccharide relative to the iron salts and the reaction temperature) and added functionalizations (amino groups and fluorophore) on the properties of the particles relevant for their use as contrast agents in magnetic resonance and optical imaging. 


\section{EXPERIMENTAL}

\section{Reagents}

High-purity water (resistivity of 18.6 M $\Omega$ ) was employed in all synthesis. The reagents used were all of analytical grade, acquired from the following brands: a) Merck (hydrochloric acid (37\%), ethanol, potassium bromide, sodium citrate, sodium chloride, ferrous and ferric chloride, ammonium hydroxide (25\%), sodium hydroxide, hydrogen peroxide (30\%) and sodium bicarbonate); b) SigmaAldrich (epichlorohydrin and iron standards); c) Pharmacosmos (polysaccharide dextran T10 with average molecular weight of 10.000 Da); d) G\&E Healthcare (Cy5.5 NHS ester fluorophore).

\section{Synthesis of nanoparticles}

The synthesis of aminated crosslinked iron oxide nanoparticles $\left(\mathrm{CLIO}-\mathrm{NH}_{2}\right)$ was performed by coprecipitation of iron salts in alkaline medium, adapting a protocol proposed by Wunderbaldinger and Palmacci. ${ }^{32,36}$ Briefly, $\mathrm{FeCl}_{3} \cdot 6 \mathrm{H}_{2} \mathrm{O}$ was added to ice-cold dextran solution $(0.9 \mathrm{mmol}$ in acid $\mathrm{pH})$ in constant magnetic stirring for 30 minutes, under $\mathrm{N}_{2}$ atmosphere. Then, the $\mathrm{FeCl}_{2} \cdot 4 \mathrm{H}_{2} \mathrm{O}$ was added to the mixture, which was inserted in a reflux system. A volume of $10 \mathrm{~mL}$ of cooled $\mathrm{NH}_{4} \mathrm{OH}$ was added to the mixture and the reaction was conducted at either $80{ }^{\circ} \mathrm{C}$ or $60{ }^{\circ} \mathrm{C}$ for $1.5 \mathrm{~h}$. The solution of nanoparticles was centrifuged in amicon tubes (50k MWCO) to remove the remaining free dextran. In order to analyse the influence of dextran and iron salt masses and reaction temperature, different batches were produced. These syntheses were labelled A to F, and their parameters are summarized in Table 1. For comparison purposes, a batch of uncoated iron oxide nanoparticles was also prepared.

After the basic nanoparticle preparation, an additional crosslinking step of the dextran shell was made, adding a solution of $35 \mathrm{~mL}$ of $5 \mathrm{~mol} \mathrm{~L}^{-1} \mathrm{NaOH}$ and $14 \mathrm{~mL}$ of epichlorohydrin into the nanoparticles solution, under magnetic stirring for $10 \mathrm{~h}$. Finally, for the amination of the coating, $60 \mathrm{~mL}$ of $\mathrm{NH}_{4} \mathrm{OH}$ was added to the solution and maintained under magnetic stirring for a period of $24 \mathrm{~h}$. The ammonia excess was extracted by dialysis in a water bath, using Spectra/Por ${ }^{\circledR}$ membranes and changing the deionized water every 30 minutes. At the end, the solution of nanoparticles was centrifuged in amicon tubes (50k MWCO) for 15 minutes and washed several times to eliminate undesired residues. The final solution (typically $\sim 20 \mathrm{~mL}$ with an iron concentration of $10 \mathrm{mg}$ $\mathrm{mL}^{-1}$ ) was stored in a sodium citrate/sodium chloride buffer solution, at $\mathrm{pH} 8-8.3$, and kept in a refrigerator at $4{ }^{\circ} \mathrm{C}$.

The crosslinking and amination steps were performed for all synthesis, except for the batch $\mathrm{C}$ (no amination and crosslinking). A sample from batch $\mathrm{D}$ was also conjugated to the fluorescent molecule Cy5.5 via the amino groups of the NPs and the carboxyl groups present in the dye (NHS). ${ }^{42,43}$ These nanoparticles were prepared from $1 \mathrm{~mL}$ of the stock solution with the addition of $0.5 \mathrm{mmol} \mathrm{L}-1$ sodium bicarbonate solution at $\mathrm{pH} 9$. The mixture was added directly in the dye flask and stirred for $7 \mathrm{~h}$. The solution was then filtered in a separation column (Sephadex ${ }^{\mathrm{TM}} \mathrm{G}-25 \mathrm{M}$ ) with a sodium citrate/ sodium chloride buffer solution. The Cy5.5 conjugated NP batch (named D-Cy5.5) was kept at $4{ }^{\circ} \mathrm{C}$ and covered with aluminum foil to avoid photobleaching.

\section{Characterization}

The total iron concentration of the nanoparticle dispersions was determined by light absorption at $410 \mathrm{~nm}$ in a UV-Vis spectrophotometer (Lambda 35, Perkin Elmer), ${ }^{36}$ based on a calibration curve obtained from standard iron oxide samples. The morphology and size of the nanoparticles were analysed by scanning electron microscopy (SEM - EVO LS150, ZEISS) and transmission electron microscopy (TEM - JEM-2010 $200 \mathrm{kV}$, JEOL). Before collecting the samples for microscopy, the stock solution was sonicated at $40 \mathrm{kHz}$ for 10 minutes and vortexed. For TEM, all the batches were prepared by dripping a diluted solution of the nanoparticles on a TEM grid. For the SEM analysis, a drop of the diluted dispersion was spread onto a silicon substrate and subsequently covered with a thin layer of gold. The size distribution and zeta potential of the nanoparticles in aqueous dispersions were obtained in a Zetasizer ZEN3600, Malvern. The crystallinity was measured in a X-ray diffractometer (XRD - Shimadzu 7000), using Cu $\mathrm{K}_{\alpha}$ radiation $(\lambda=0.1540 \AA)$. The chemical structure was characterized by infrared spectroscopy (FTIR- Spectrum One, Perkim Elmer) in the spectral range of $4000-400 \mathrm{~cm}^{-1}$. All the samples for FTIR were freeze-dried (lyophilizer LS 3000, Terroni) and the pellets were prepared with a mass ratio of $10 \%$ of nanoparticles to $90 \%$ of $\mathrm{KBr}$. Thermogravimetric analysis (TA Instruments Q 600) was performed in a nitrogen atmosphere with a heating rate of $20{ }^{\circ} \mathrm{C} \min ^{-1}$ up to a final temperature of $900^{\circ} \mathrm{C}$. The magnetization curves were obtained in a vibrating sample magnetometer (VSM - EZ9) with the freezedried samples.

Measurements of the magnetic relaxation times T1, T2 and relaxivity of the nanoparticle dispersions were performed in a clinical 1.5 T MRI equipment ( $450 \mathrm{G} \& \mathrm{E}$ ). A special phantom made of a plastic foam was build for the MRI measurements (see Figure 7 for an image of the phantom). Up to 16 syringes can be disposed symmetrically around the central axis of the phantom. Five dilutions were prepared for each type of nanoparticle: $\mathrm{C}_{0} / 100, \mathrm{C}_{0} / 200, \mathrm{C}_{0} / 500, \mathrm{C}_{0} / 1000 \mathrm{e}$ $\mathrm{C}_{0} / 2000$, where $\mathrm{C}_{0}$ is the stock solution concentration. Syringes with $1 \mathrm{~mL}$ of each dilution were then inserted in the phantom for imaging, together with a larger volume (placed in the middle of the phantom) of citrate buffer, used as a reference material. The data were acquired with a skull coil in three slices of the phantom using fast spin echo (FSE) and fast spin echo inversion recovery (FSE-IR) sequences to obtain, respectively, T2 and T1 values of the NPs suspensions. FSE

Table 1. Synthesis parameters for the batches labelled A to F. The table shows the mass of the major components, the reaction temperature and reflux time. The steps of dextran crosslinking and amination are the same for all batches (see text), except for batch $\mathrm{C}$ where amination and crosslinking were not employed

\begin{tabular}{|c|c|c|c|c|c|}
\hline Batch & Dextran mass $(\mathrm{g})$ & $\mathrm{Fe}^{+3}$ mass $(\mathrm{g})$ & $\mathrm{Fe}^{+2}$ mass $(\mathrm{g})$ & Temperature $\left({ }^{\circ} \mathrm{C}\right)$ & Reflux time (h) \\
\hline A & 9 & 0.65 & 0.40 & 80 & 1.5 \\
\hline B & 9 & 0.65 & 0.40 & 60 & 1.5 \\
\hline $\mathrm{C}$ & 9 & 0.65 & 0.40 & 80 & 3.0 \\
\hline $\mathrm{D}$ & 4.5 & 0.65 & 0.40 & 80 & 1.5 \\
\hline E & 2.5 & 0.65 & 0.40 & 80 & 1.5 \\
\hline $\mathrm{F}$ & 2.5 & 1.3 & 0.80 & 80 & 1.5 \\
\hline
\end{tabular}


images were acquired with a slice thickness of $2.0 \mathrm{~mm}$, TR (time to repetition) of $1000 \mathrm{~ms}$, echo train length of 3 and effective echo times TE varying from 7.4 to $250 \mathrm{~ms}$. For FSE-IR sequences, TR was set at $3000 \mathrm{~ms}$, echo train length of 10 and inversion times TI varied from 50 up to $1300 \mathrm{~ms}$, with $\mathrm{TE}=21 \mathrm{~ms}$.

\section{RESULTS AND DISCUSSION}

\section{Synthesis and general characteristics of the nanoparticles}

In the following, we present the properties of the produced composite nanoparticles, emphasizing differences resulting from the synthesis routes labelled A to F (Table 1). An overview of the structural characteristics, size, and morphology of the nanoparticles from different batches are shown in Figure 1. Nanoparticles from batch A (referred to as the standard procedure) appear in the TEM micrographs (Figure 1A) as rounded structures representing the iron oxide core. Their average mean core diameter was $9.3 \pm 1.6 \mathrm{~nm}$ and the mean hydrodynamic diameter in aqueous solutions was $16.2 \pm 7.4 \mathrm{~nm}$. EDS was also performed and confirmed the presence of the elements $\mathrm{Fe}$ and $\mathrm{O}$ in the nanoparticles (data not shown). The crystallinity of the nanoparticles can be seen from the well-defined lattice planes in the high-resolution TEM image of Figure $1 \mathrm{~B}$ and in the x-ray diffractograms shown in Figure 1E. The XRD pattern of nanoparticles for all the batches reveal distinct peaks at $2 \theta$ equal to $30.3^{\circ}, 35.6^{\circ}, 43.0^{\circ}, 57.2^{\circ}$ and $62.7^{\circ}$, representing the crystal planes (220), (311), (400), (511), and (440) of spinel ferrite ( ${ }^{\circ} 5-628$
JCPDS). The average crystal size corresponding to the (311) peak was calculated according to the Scherrer equation: $D=K \lambda / \beta \cos (\theta)$; where $\mathrm{K}$ is a constant equal to $0.94, \lambda$ is the wavelength of $\mathrm{X}$-ray, $\beta$ is the full width at half maximum of the Bragg peak. ${ }^{44}$ The average size of the nanoparticles for the different synthesis is given in Table 2 . This includes estimations obtained from TEM, XRD and DLS. As TEM images give the particle size of the core alone, without the contribution of the dextran coating, the sizes extracted from TEM observations are smaller than the hydrodynamic radius obtained from DLS, as expected.

It was observed that changing the reaction temperature from $80{ }^{\circ} \mathrm{C}$ to $60{ }^{\circ} \mathrm{C}$ (batch B), increased the nanoparticles size by $\sim 32 \%$ measured by DLS. Nanoparticles from batch C, were smaller than for the other batches (the mean hydrodynamic diameter was around $13.8 \mathrm{~nm}$ ), most probably because of the absence of crosslinking and amination steps in the reaction. Halving the mass ratio of dextran to iron salts in relation to the batch A, resulted only in a small increase in the nanoparticles mean diameter (batch D). However, further decrease of the mass ratio of dextran in the batch E (reaching $25 \%$ of the value used in A) resulted in nanoparticles with a larger core (see Table 2 and Figure 1B). The mean hydrodynamic diameter also increased $(48.5 \mathrm{~nm})$, indicating a more pronounced particle aggregation. Decreasing even further the dextran to iron mass ratio in the batch F (the content of iron salts was twice that for the batch E) resulted in very large particles or aggregates, most of which precipitated in the reaction vessel. The size distribution of batch $F$ shown in Figure 1D represents only the fraction of particles that
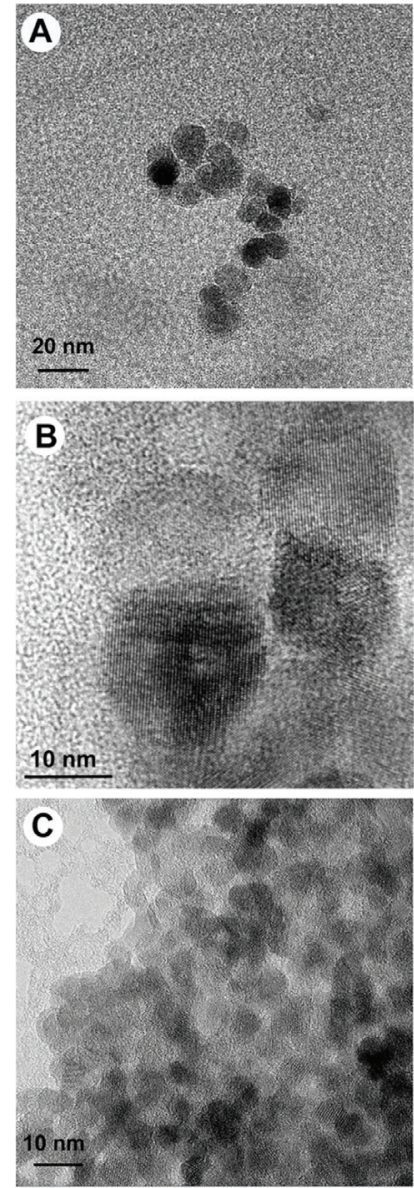

D

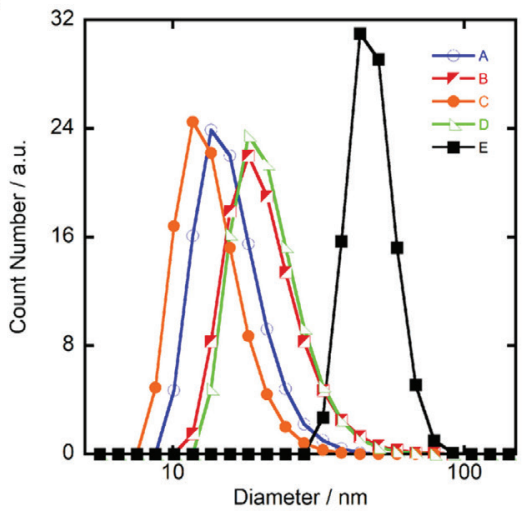

E

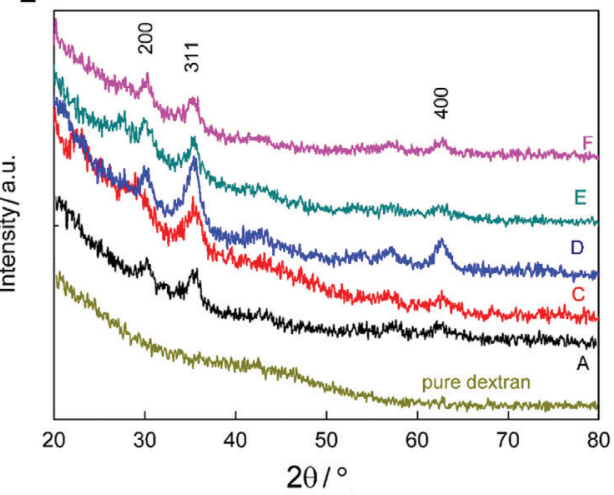

Figure 1. TEM micrographs of samples from $(A)$ batch $A,(B)$ batch $E$, and $(C)$ batch $F$. (D) Size distribution by number of nanoparticles from different batches obtained by DLS, (E) X-ray diffractograms of nanoparticles coated with dextran for batches A to F. A measurement of pure dextran powder is also added for comparison 
Table 2. Average size of the nanoparticles for different batches obtained by DLS, TEM and XRD

\begin{tabular}{cccc}
\hline \multirow{2}{*}{ Synthesis } & $\begin{array}{c}\text { Mean hydrodynamic } \\
\text { diameter }(\mathrm{nm})\end{array}$ & \multicolumn{2}{c}{ Iron oxide core $(\mathrm{nm})$} \\
\cline { 2 - 4 } & DLS & TEM & XRD \\
\hline A & $16.2 \pm 7.4$ & $9.3 \pm 1.6$ & 7.3 \\
B & $21.4 \pm 6.8$ & - & - \\
C & $13.8 \pm 3.9$ & - & 5.7 \\
D & $21.8 \pm 6.7$ & - & 6.3 \\
E & $48.5 \pm 8.9$ & $17 \pm 2$ & 10.5 \\
F & $196 \pm 41.2$ & $6 \pm 1$ & 8.8 \\
\hline
\end{tabular}

remained dispersed, showing a hydrodynamic diameter around 196 $\mathrm{nm}$. This value however reflects the agglomeration of nanoparticles in aqueous media and not their individual size. Indeed, TEM images of nanoparticles from batch $\mathrm{F}$ show individual particles with diameters around $6 \mathrm{~nm}$, but disposed in large agglomerates (Figure 1C). For small amounts of dextran, the polymer molecules most probably bind several adjacent iron oxide particles.

The chemical groups present in the composite nanoparticles can be identified in Figure 2, where FTIR spectra of samples from batches A, E, and of the pure dextran are given. The peak at $\sim 593 \mathrm{~cm}^{-1}$, corresponding to the stretching mode of $\mathrm{Fe}-\mathrm{O}$ bonds from the iron oxide, appears in all batches. ${ }^{8,19}$ The characteristic dextran peaks are also seen: the stretching of $\mathrm{OH}$ groups (broad peak at $3410 \mathrm{~cm}^{-1}$ ); the $\mathrm{CH} / \mathrm{CH}_{2}$ absorption band at $2922 \mathrm{~cm}^{-1}$; the $\mathrm{C}-\mathrm{O}$ band at $1110 \mathrm{~cm}^{-1}$; the deformation mode of the $\alpha$-glucopyranose ring at 768 and $952 \mathrm{~cm}^{-1}$, and the $\mathrm{C}=\mathrm{O}$ stretching at $\sim 1644 \mathrm{~cm}^{-1} .^{8,10,21,23}$ The absorption band at $1464 \mathrm{~cm}^{-1}$ is attributed to the angular deformation of $\mathrm{N}-\mathrm{H}$ bond of the amine groups present in the nanoparticles shell. ${ }^{19}$

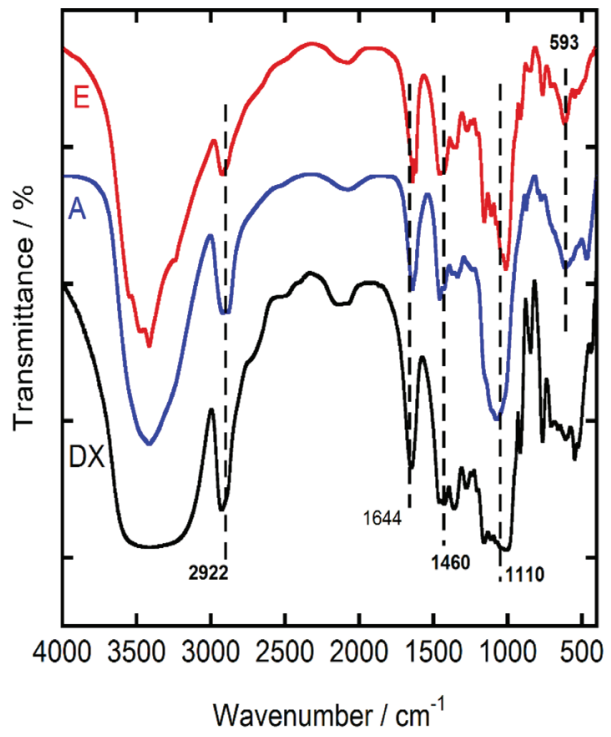

Figure 2. FTIR spectra of pure dextran and of nanoparticles from batches $A$ and $E$

To confirm the incorporation of the fluorophore, UV-Vis and fluorescence spectroscopy was performed. Figure 3 shows the UV-Vis and fluorescence spectrum of the nanoparticles from batch D labeled with Cy5.5. It is observed the absorption peak around $679 \mathrm{~nm}$ and emission peak around $690 \mathrm{~nm}$ characteristic of the dye, confirming conjugation of the fluorophore in the nanoparticle structure. . $^{42,43}$

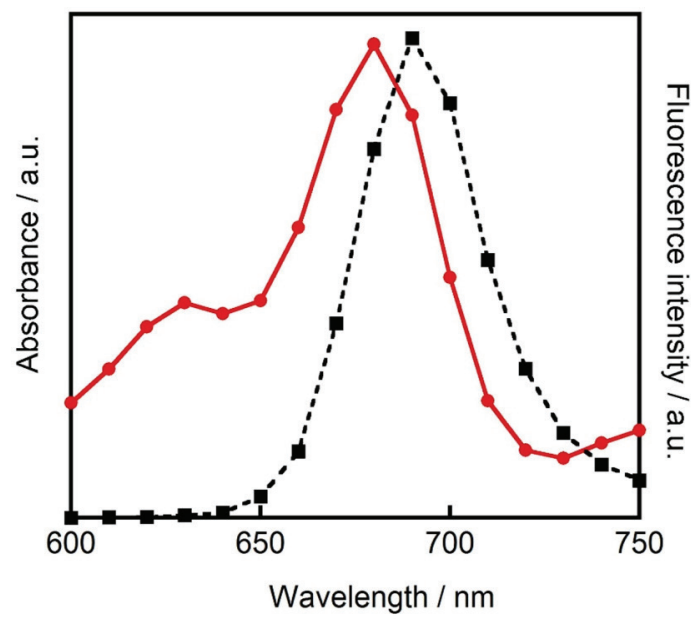

Figure 3. Absorption (-) and emission ( $\mathbf{\square}$ ) spectra of nanoparticles of batch D labelled with Cy5.5

\section{Colloidal stability}

Figure 4 show the hydrodynamic diameter, polydispersity index, and zeta potential of the nanoparticles from batches A, C, and D at various $\mathrm{pHs}$. The mean hydrodynamic diameter varied very little, with no sign of flocculation, indicating good size stability against large $\mathrm{pH}$ variations. The behavior for the nanoparticles from batch $\mathrm{B}$ was similar. However, samples from batches $\mathrm{E}$ and $\mathrm{F}$ showed tendency to agglomerate, mainly in a $\mathrm{pH}$ range around 6 - 8 (data not shown). The zeta potential $(\zeta)$ of the nanoparticles from batch $\mathrm{A}$ in aqueous solution at $\mathrm{pH} \sim 6.5$ was $+5.8 \pm 0.5 \mathrm{mV}$. The positive values of zeta potential are consistent with the presence of amino groups on the NPs surface and is an indication that the amination process was effective. In fact, for the batch $\mathrm{C}$, where no amination step was employed, $\zeta=$ $-6.5 \pm 0.7 \mathrm{mV}$ at $\mathrm{pH} \sim 8$. In this case, the negative potential arises from the presence of hydroxyl groups on the surface of the dextran shell. It was also observed that for the cross-linked samples (A, D and E), the isoeletric point were around $\mathrm{pH}=8$ and for sample $\mathrm{C}$ was around 4.5 (Figure 4B), confirming the modification of the surface of the particle by the amination process. In all cases above, the absolute values of the zeta potential however were relatively low, compared to the value of the naked iron oxide nanoparticles $(-41.4 \mathrm{mV}$ ) (data not shown), indicating that the particles in the dispersion are actually stabilized mostly by steric repulsion due to the dextran coating. ${ }^{9,14,20}$

\section{Thermal Analysis}

Thermal gravimetric analysis (TGA) was employed to access information on the mass fraction of different components of the particles: the iron core, the dextran shell and other volatile residues. Figure 5 shows the weight loss curve of lyophilized nanoparticles from different batches as a function of temperature, including also curves for pure dextran and naked iron oxide nanoparticles as references. Different stages of mass loss were observed in the thermograms. The first stage at $\mathrm{T} \sim 100{ }^{\circ} \mathrm{C}$ is attributed to the evaporation of water molecules contained in the system. This corresponds to a weight fraction below $\sim 7 \%$ for all batches. The second step at around $250-300{ }^{\circ} \mathrm{C}$ is attributed to the onset of thermal degradation of the dextran shell. ${ }^{10,19}$ This onset temperature is especially low for batch C, where no crosslinking step was applied. Interestingly, in this case, the association with iron oxide caused a decrease in the initial degradation temperature, compared to pure dextran. For all other batches, where dextran was crosslinked, the onset degradation temperature is shifted to higher temperatures (about $40^{\circ} \mathrm{C}$ ). 
A

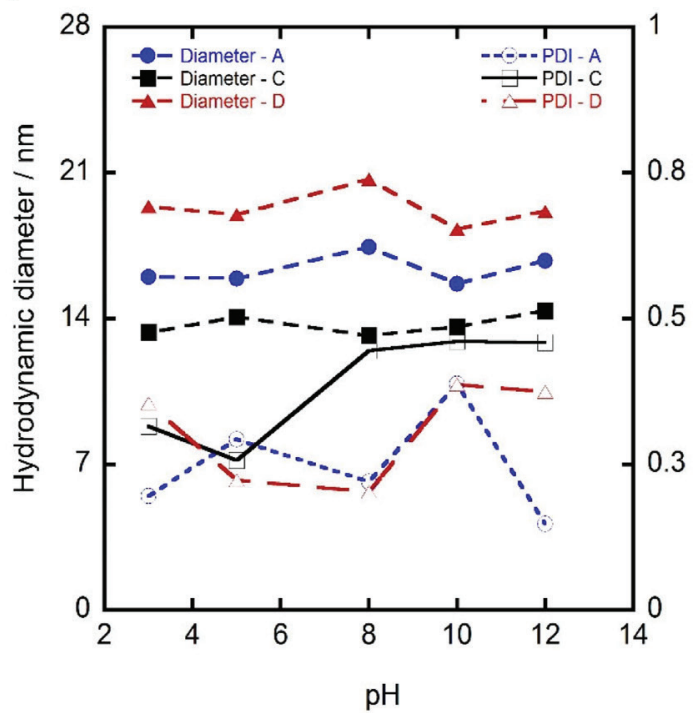

B

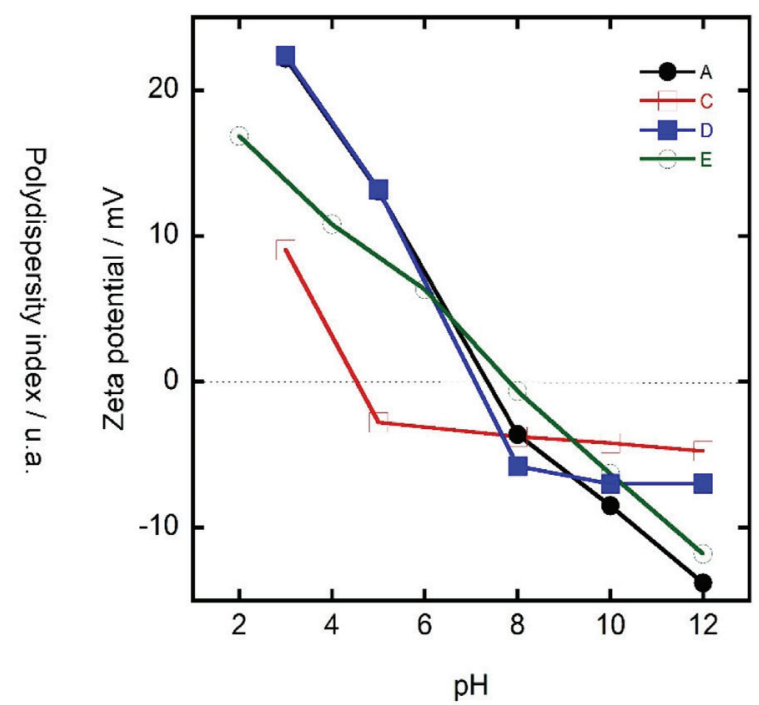

Figure 4. (A) Average diameter and polydispersity index, and (B) zeta potential of nanoparticles from different batches as a function of pH

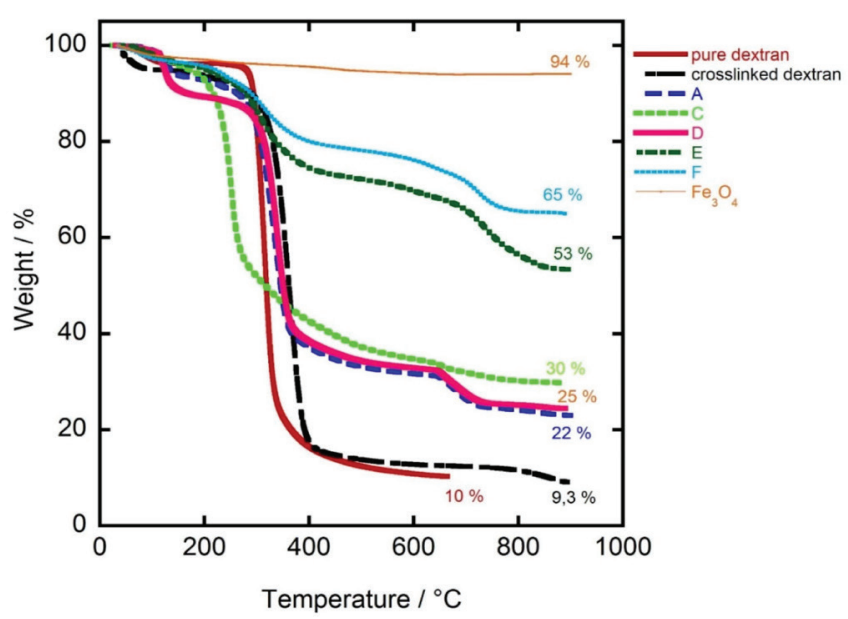

Figure 5. Mass loss as a function of temperature for nanoparticles from batches $A, C, D, E$, and $F$. The thermograms for naked iron oxide nanoparticles, pure dextran and crosslinked dextran are also shown for comparison

A third stage of mass loss is observed around $650{ }^{\circ} \mathrm{C}$ possibly related to the final degradation of the organic residues more tightly bound to the nanoparticles. ${ }^{8}$ This stage is particularly pronounced for the batches that included the crosslinking step. However, for particles with a larger content of dextran (batches $\mathrm{A}$ and $\mathrm{D}$ ) the degradation temperature of the third stage is $\sim 80{ }^{\circ} \mathrm{C}$ lower as compared to the composite systems with a low dextran content (batches $\mathrm{E}$ and F), meaning that excess of dextran reduce the thermal stability of the organic shell in close contact with the iron oxide surface. The final residual mass at $900{ }^{\circ} \mathrm{C}$, corresponding approximately to the iron oxide fraction by mass in the NPs is given by the percentage numbers in Figure 5. Overall, the differences in mass fraction of organic residues seen among the various batches is compatible with the different values of dextran concentration used in the synthesis.

\section{Magnetization and nuclear magnetic relaxation measurements}

Figure 6 shows the magnetization as a function of external magnetic field at room temperature for lyophilized nanoparticles. The nanoparticles from all different synthesis showed no residual magnetization at zero external magnetic field and a magnetization curve with no hysteresis, characteristics of a superparamagnetic behavior. The saturation magnetization $\left(\mathrm{M}_{\mathrm{s}}\right)$ increased from $9.0 \mathrm{emu} / \mathrm{g}$ for batch A to $43.3 \mathrm{emu} / \mathrm{g}$ from batch $\mathrm{F}$, reflecting the different proportions of iron oxide in the total mass of the samples seen in Figure 5. ${ }^{10,19}$ Indeed, estimating the effective iron oxide mass from the residual fraction at $900{ }^{\circ} \mathrm{C}$ one gets $\mathrm{M}_{\mathrm{s}}(\mathrm{F}) \sim 66,6 \mathrm{emu} / \mathrm{g}$ and $\mathrm{M}_{\mathrm{s}}(\mathrm{E}) \sim 60 \mathrm{emu} / \mathrm{g}$, which is very close to the value of the uncoated nanoparticles ( $66 \mathrm{emu} / \mathrm{g}$ ). The batches $\mathrm{A}$ and $\mathrm{D}$, obtained in the presence of a larger content of dextran, have $M_{\mathrm{s}} \sim 41 \mathrm{emu} / \mathrm{g}$. This reduction in saturation magnetization may be associated to the smaller sizes of the magnetic core of these particles and to the larger mean interparticle distance (because of the thicker coating) which reduce dipolar interactions. Reduced magnetization values have been reported for small ferrite particles. Surface spin disorder, such as the existence of a magnetically dead layer on the particle's surface, the presence of canted spins or even the diamagnetic polymer layer at the surface of nanoparticle may all contribute to a reduced magnetization. ${ }^{20,45,46}$ Small variations were observed in the shape of the magnetization curves among the various synthesis, most probably reflecting differences in the particle size distributions. Larger particles

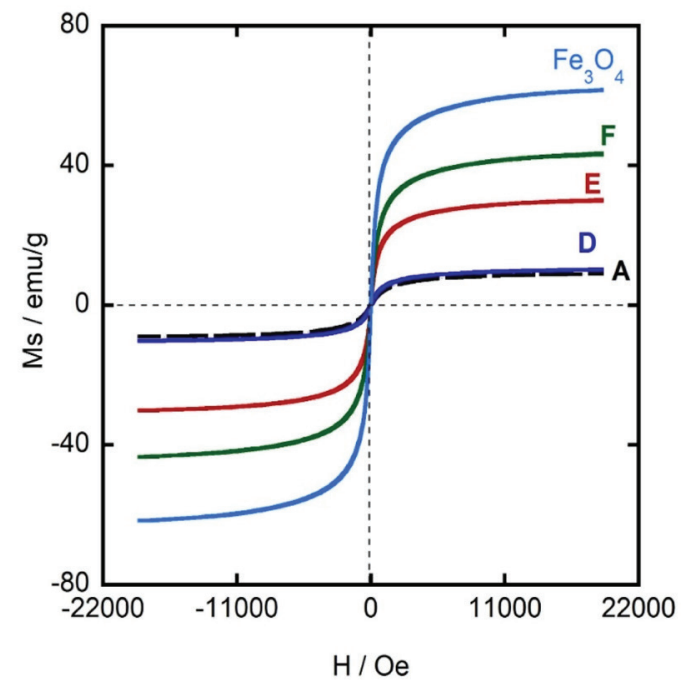

Figure 6. Magnetization as a function of applied magnetic field at room temperature for nanoparticles of batch A, C, D, E, F and pure $\mathrm{Fe}_{3} \mathrm{O}_{4}$ 
tend to magnetize more easily and this trend is clearly observed in our data. ${ }^{47}$ As we had no control of the spatial arrangement of nanoparticles (e.g. the average interparticle distances) after deposition on the sample holder used for the magnetization measurements, it is difficult at present to provide an unambiguous interpretation for the differences in the shape of the magnetization curves.

Next, we discuss nuclear magnetic relaxation measurements of aqueous solutions of nanoparticles from different batches performed at a $1.5 \mathrm{~T}$ clinical MRI equipment. A typical MRI fast spin eco image of one slice of the phantom containing syringes with nanoparticles solutions from batches $\mathrm{A}, \mathrm{C}$ and $\mathrm{D}$ at five different concentrations (dilution ratio from 1:100 to 1:2000) is shown in Figure 7A. This illustrates a typical image of the set necessary to obtain the T1 and T2 relaxation curves of the NPs suspensions. The circles correspond to cross sections of each syringe, from which the signal (or mean pixel) intensity were extracted. Figures 7B-C show curves of signal intensity $\mathrm{S}(\mathrm{t})$ as a function of TE and TI for the nanoparticles of batch A. They represent the typical behavior seen for all other NPs tested. The T2 relaxation times were extracted from fittings of Equation 1 to the data derived from the FSE sequences, as in Figure 7B:

$$
S(T E)=S_{\max } e^{-\frac{T E}{T 2}}+\mathrm{S}_{\mathrm{n}}
$$

where $S_{n}$ is the noise level in the image. Similarly, the T1 relaxation times were obtained by fitting the curve of the inversion recovery signal, as in Figure 7C:

$$
S(T I)=S_{\max }\left(1-a e^{-\frac{T I}{T 1}}+e^{-\frac{T R}{T 1}}\right),
$$

with $a$ close to 2 .

\section{B}
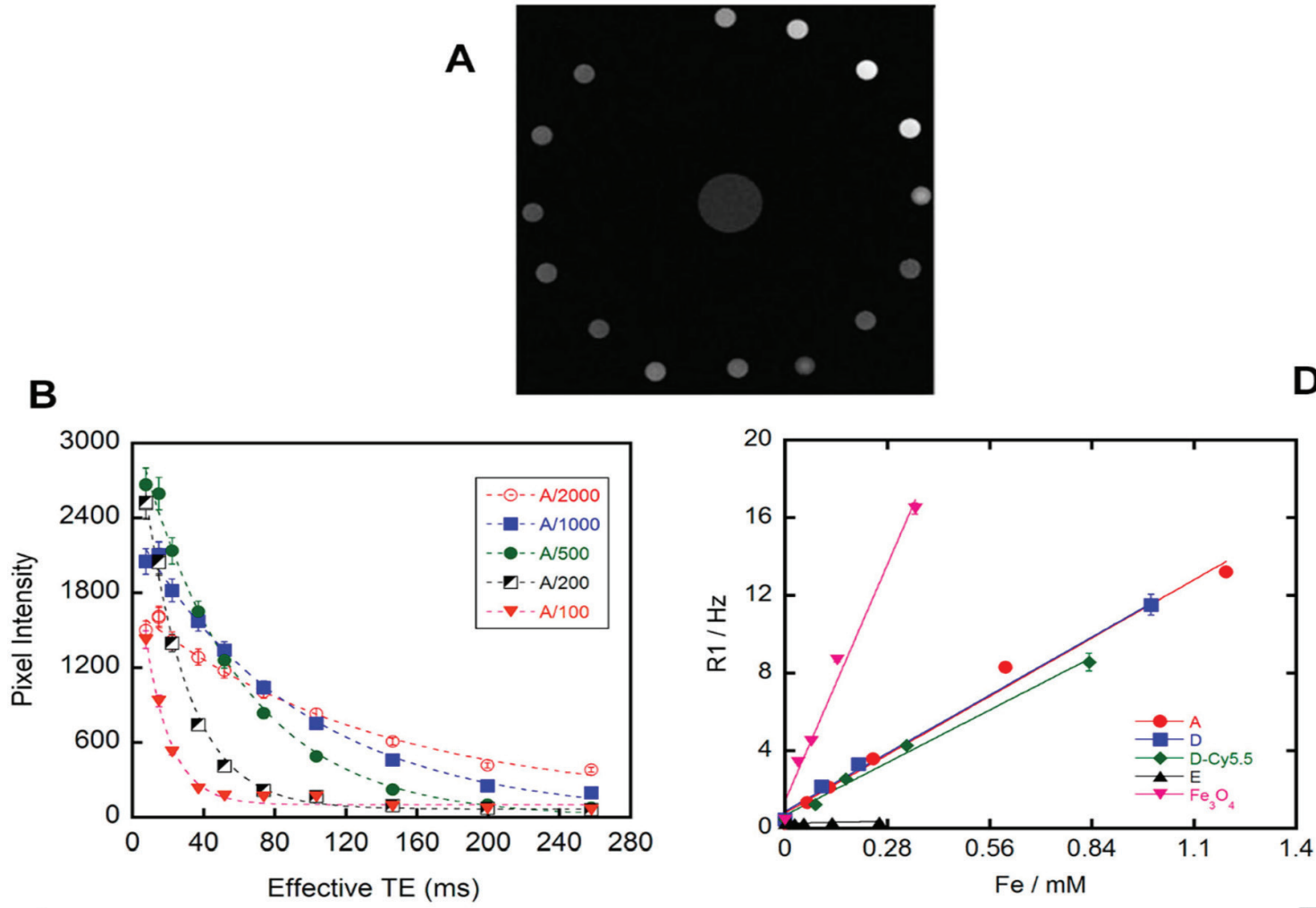

C
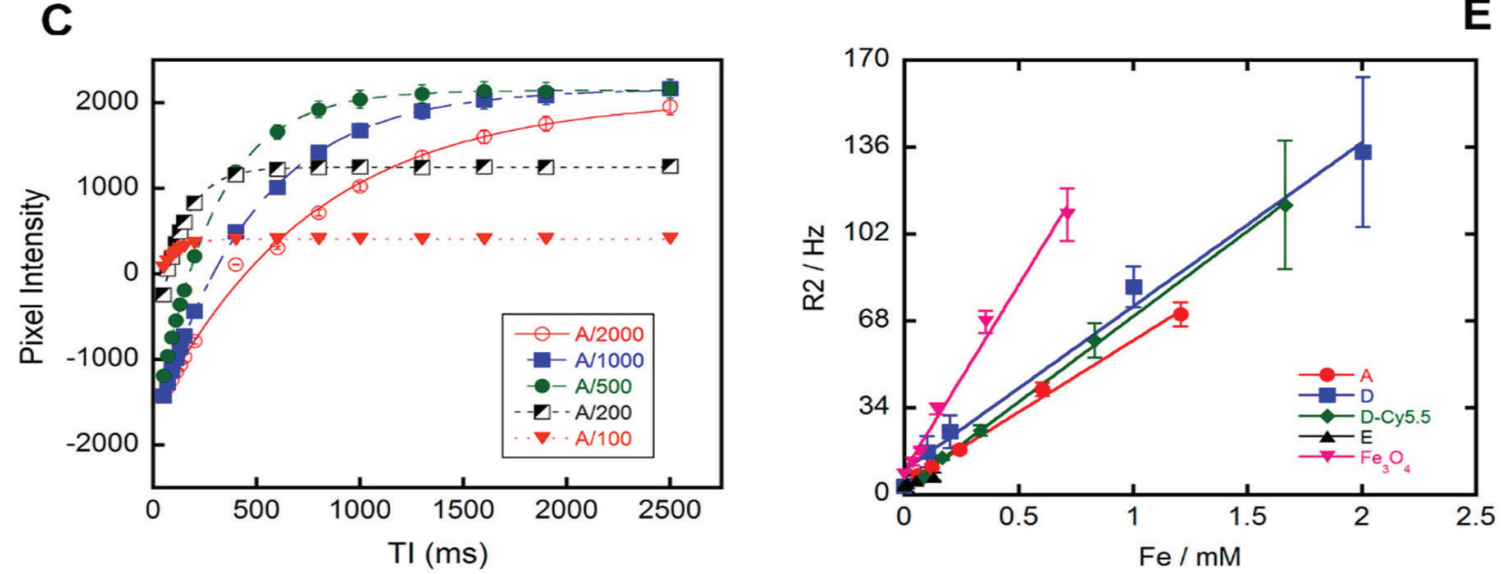

Figure 7. Nuclear magnetic relaxation measurements of aqueous dispersions of nanoparticles obtained with a 1.5 T MRI equipment. (A) Typical MRI image (FSE with $T R=1000 \mathrm{~ms}$ and $T E=7.4 \mathrm{~ms}$ ) of the phantom showing cross sections of the syringes filled with dispersions with different NP concentrations. (B) Mean pixel intensity as a function of effective TE in images recorded by FSE sequences for NPs suspensions from synthesis A. (C) Mean pixel intensity as a function of TI for aqueous suspensions with different concentrations of nanoparticles from synthesis A. The dilution ratio (100 to 2000) from the stock solution is indicated in the figure. (D) Relaxation rates $\mathrm{R} 1$ and $(E) \mathrm{R} 2$ as a function of iron concentration. Curves for synthesis $A, D, D$-Cy5.5, E and uncoated Fe ${ }_{3} \mathrm{O}_{4}$ are displayed 
The corresponding relaxation rates $\mathrm{R} 1(=1 / \mathrm{T} 1)$ and $\mathrm{R} 2(=1 / \mathrm{T} 2)$ are plotted as a function of nanoparticle concentration in Figure 7D e 7E for samples of batches A, D, D-Cy5,5, E, and naked $\mathrm{Fe}_{3} \mathrm{O}_{4}$. From these curves the relaxivities $r_{1}$ and $r_{2}$ (the relaxation rate per unit concentration of the contrast agent) were extracted. The uncoated iron oxide NPs presented the largest relaxivity values $\left(r_{2}=148 \pm 5 \mathrm{~L} \mathrm{mmol}^{-1} \mathrm{~s}^{-1}\right.$, and $\left.\mathrm{r}_{1}=47 \pm 3 \mathrm{~L} \mathrm{mmol}^{-1} \mathrm{~s}^{-1}\right)$, i.e, they have the highest in vitro efficiency of contrast change. The absence of a coating allows close proximity of diffusing water protons to the magnetic field of the NPs. This, together with the large saturation magnetization, enhances the decay of spin coherence and spin-lattice interactions, accelerating nuclear spin relaxation. ${ }^{48,49}$

For the batches A to D of dextran-coated particles, the values of $r_{1}$ were within 10 to $11 \mathrm{~L} \mathrm{mmol}^{-1} \mathrm{~s}^{-1}$, including the one with the addition of the fluorophore Cy5.5. Similarly, $\mathrm{r}_{2}$ values were also close among the different syntheses $\left(\sim 58-68 \mathrm{~L} \mathrm{mmol}^{-1} \mathrm{~s}^{-1}\right)$, even when reducing the dextran content by $50 \%$ (what also resulted in changes in hydrodynamic radius of $\sim 35 \%$ ). The ratio of the relaxivities $r_{2} / r_{1}$ obtained for the dextran-coated particles from batches A to D were around 5, within the range found for nanoparticle systems classified as USPION (ultrasmall superparamagnetic iron oxide contrast agents). ${ }^{20}$ Particles from batch E synthesized in the presence of $75 \%$ less dextran content compared to the standard procedure (A), induced very small $r_{1}$ values, and $r_{2} \sim 27 \mathrm{~L} \mathrm{mmol}^{-1} \mathrm{~s}^{-1}$. It is at present unclear the reason of such behavior, which seems to be related to the poor stability and higher aggregation of such nanoparticles. In fact, it has been proposed that clustering of nanoparticles weakens the effect on the acceleration of T1 relaxation. ${ }^{50}$ Particles from batch F (with even higher reductions of dextran content during the synthesis) were too unstable to allow MRI measurements to be performed.

\section{CONCLUSIONS}

Superparamagnetic nanoparticles were successfully synthesized by the co-precipitation method of iron salts in alkaline medium with a core of crystalline iron oxide with average diameters around $9-17 \mathrm{~nm}$ and average hydrodynamic diameters of $16-50 \mathrm{~nm}$. We investigated the effect of changes in the synthesis parameters on the size and properties of the composite nanoparticles. Changes in temperature had only a little influence on the size distribution and the variation in the ratio of dextran proved to be the most effective parameter for controlling the size of the nanoparticles. The dextran content also impacted the thermal stability of the coating, with the particles having the largest dextran content showing lower degradation temperatures. The zeta potentials values of all batches showed a positive net surface charge, except for the batch for which the amination step was not performed and surface termination with hydroxyl groups predominate. The magnetization curve of the nanoparticles showed no residual magnetization when the magnetic field was removed and no hysteresis, indicating superparamagnetic behavior. The nuclear magnetic relaxation times of water protons in aqueous suspensions for different batches of nanoparticles did not show major differences, with no significant effect due to the addition of fluorophore or changes in the dextran fraction up to 50\%. Larger reductions in the ratio of dextran to iron salts resulted in particles with slightly larger sizes, higher saturation magnetization and distinct nuclear magnetic relaxation times.

\section{ACKNOWLEDGEMENTS}

The authors would like to acknowledge CNPq and HewlettPackard for financial support, the Magnetism Laboratory (LAM) of the Physics Institute/UFRGS for assistance in the magnetization measurements and Dr J. R. Hoefel for granting the access to the MRI equipment of the CDI Center at São Lucas Hospital, PUCRS.

\section{REFERENCES}

1. Khalafalla, S.; E.; Reimers, G. W.; IEEE Trans. Magn. 1980, 16, $2,2$.

2. Schnepp, Z.; Wimbush, S. C.; Antonietti, M.; Giordano, C.; Chem. Mat. 2010, 22, 5340 .

3. An, H.; Ahn. H. J.; Mater. Lett. 2012, 81, 41.

4. Frey, N.; A.; Peng, S.; Cheng, K.; Sun, S.; Chem. Soc. Rev. 2009, 38, 2532.

5. Qasem, R. A. M.; Gedanken, A.; J. Colloid Interface Sci. 2005, 284, 489.

6. Tourinho, F. A.; Franck, R.; Massart, R.; J. Mater. Sci. 1990, 25, 3249.

7. Massart, R.; Dubois, E.; Cabuil, V.; Hasmonay, E.; J. Magn. Magn. Mater. 1995, 149, 41030.

8. Bautista, M. C.; Miguel, O. B.; Morales, M. O.; Serna, C.; Verdaguer, S. V.; J. Magn. Magn. Mater. 2005, 293, 20.

9. Bhattacharya, D.; Sahu, S. K.; Banerjee, I.; Das, M; Mishra, D.; Maiti, T. K.; Pramanik, P.; J. Nanopart. Res. 2011, 13, 4173.

10. Easo, S. L.; Mohanan. P. V.; Carbohydr. Polym. 2013, 92, 726.

11. Rosen, J. E.; Chan, Y.; Shieh, D. B.; Gu, F. X.; NanomedicineNanotechnology 2012, 8, 275.

12. Jansch, M.; Stumpf, P.; Graf, C.; Ruhl, E.; Muller, R. H.; Int. J. Adv. Pharm. 2012, 428, 125.

13. Lee, S. Y.; Ahn, C. Y.; Lee, J.; Chang, J. H.; Talanta 2012, 93, 160.

14. Qiao, R.; Yang, C.; Gao, M.; J. Mater. Chem. 2009, 19, 6274.

15. Schladt, T.; Schneider, K.; Schild, H.; Tremel, W.; Dalton Trans. 2011, 40,6315 .

16. Figuerola, A.; Corato, R. D.; Manna, L.; Pellegrino, T.; Pharmacol. Res. 2010, 62, 126 .

17. Yang, H.; Zhuang, Y.; Sun, Y.; Dai. A.; Shi, X.; Wu, D.; Li, F.; Hu, H.; Yang, S.; Biomaterials 2011, 32, 4584.

18. Singh, R.; Lillard, J. R. J. W.; Exp. Mol. Pathol. 2009, 86, 3, 215.

19. Hong, R. Y. J.; Li, H.; Qu, J. M.; Chen, L. L.; Li, H. Z.; Chem. Eng. J. 2009, 150, 572 .

20. Laurent, S.; Forge, D.; Port, M.; Roch, A.; Robic, C.; Elst, L. V.; Muller, R. N.; Chem. Soc. Rev. 2008, 108, 2064.

21. Mahmoudi, M. S.; Wang, B.; Laurent, S.; Sem, T.; Adv. Drug Delivery Rev. 2011, 63, 24.

22. Hayat, M. A.; Cancer Imaging: Lung and Breast Carcinomas, Elsevier Academic Press: UK, 2008, cap. 2.4.

23. Hong, R. Y.; Feng, B.; Chen, L. L.; Liu, G. H.; Li, H. Z.; Zheng, Y.; Wei, D.; Biochem. Eng. J. 2008, 42, 290.

24. Kim, E. H.; Ahn. Y.; Lee, H. S.; J. Alloy Compd. 2007, 434, 633.

25. Juang, J. H.; Wang. J. J.; Shen, C. R.; Kuo, C. H.; Chien, Y. W.; Kuo, H. Y.; Chen, F. R.; Chen, M. H.; Yen, T. C.; Tsai, Z. T.; Transplant. Proc. 2010, 42, 2104.

26. Muthiah, M.; Park, I.; Cho, C.; Biotechnol. Adv. 2013, 31, 8, 1124.

27. Pouliquen, D.; Jeune, J. J. L.; Perdrisot, R.; Ermias, A.; Jallet, P.; Magn. Reson. Imaging 1991, 9, 275.

28. Josephson, L.; Tung, C. H.; Moore, A.; Weissleder, R.; Bioconjugate Chem. 1999, 10, 186.

29. Hradil, J.; Pisarev, A.; Babic, M.; Horák, D.; China Particuol. 2007, 5, 162.

30. Ravikumar, C.; Kumar, S.; Bandyopadhyaya, R.; Colloid Surf., A 2012, $403,1$.

31. Tassa, C.; Shaw, S. Y.; Weissleder, R.; Acc. Chem. Res. 2011, 44, 842.

32. Palmacci, S.; Josephson, L.; US Pat. 5,262,176 1993.

33. Liu, G.; Hong, R. Y.; Guo, L.; Li, Y. G.; Li, H. Z.; Appl. Suf. Sci. 2011, 257, 6711 .

34. Yu, M.; Huang, S.; Yu, K. J.; Clyne, A. M.; Int. J. Mol. Sci. 2012, 13, 5554. 
35. Liu, D. Wu, W.; Ling, J.; Wen, S.; Gu, N.; Zhang, X.; Adv. Funct. Mater. 2011, 21, 1498 .

36. Wunderbaldinger, P.; Josephson, L.; Weissleder, R.; Academic Radiology 2002, 9, S304.

37. Lee, N. Yoo, D.; Ling, D.; Cho, M. H.; Hyeon, T.; Cheon, J.; Chem. Soc. Rev. 2015, 115, 10637

38. Yen, S. K., Padmanabhan, P.; Selvan, S. T.; Theranostics 2013, 3, 986.

39. Cho, W. S.; Cho, M.; Kim, S. R.; Choi, M.; Lee, J. Y.; Han, B. S.; Park, S. N.; Yu, M. K.; Jon, S.; Jeong, J.; Toxicol. Appl. Pharmacol. 2009, 239, 106.

40. Cha, E. J.; Jang, E. S.; Sun, I. C.; Lee, I. J.; Ko, J. H.; Kim, Y. I.; Kwon, I. C.; Kim, K.; Ahn, C. H.; J. Controlled Release 2011, 155, 152.

41. McCarthy, J.; Weissleder, R.; Adv. Drug Delivery Rev. 2008, 60, 1241.

42. Josephson, L.; Kircher, M. F.; Mahmood, U.; Tang, Y.; Weissleder, R.; Bioconjugate Chem. 2002, 13, 554.

43. Medarova, Z.; Evgenov, N. V.; Dai, G.; Weir, S. b.; Moore, A.; Nat. Protoc. 2006, 1, 429.
44. Cullity, D.; Elements of X-ray Diffraction, Addison-Wesley Publishing Co. Inc: USA, 1967, cap. 3.

45. Lu, A.; Salabas, E. L.; Schüth, F.; Angew. Chem. 2007, 46, 1222.

46. Respaud, M.; Broto, J. M.; Rakoto, H.; Fert, A. R.; Thomas, L.; Barbara, B.; Verelst, M.; Snoeck, E.; Lecante, P.; Mosset, A.; Osuna, J.; Ely, T. O.; Amiens, C.; Chaudret, B.; Phys. Rev. B: Condens. Matter Mater. Phys. 1998, 57, 2925.

47. Russier, V.; Montferrand, C.; Lalatonne, Y.; Motte L.; J. App. Phys. 2012, 112, 073926-1

48. Vuong, Q. L.; Gillis, P.; Roch, A.; Gossuin, Y.; Wiley Interdiscip. Rev.: Nanomed. Nanobiotechnol. 2017, 9, 1.

49. Demas, V.; Lowery, T. J.; New J. Phys. 2011, 13, 025005.

50. Roch, A.; Goussuin, Y.; Muller, R. N.; Gills, P.; J. Magn. Magn. Mater. 2005, 293, 532 . 